\title{
Re: Subramanian and Kumar, Increases in COVID-19 are unrelated to levels of vaccination across 68 countries and 2947 counties in the United States
}

\author{
Andreas Backhaus ${ }^{1}$ \\ ${ }^{1}$ Federal Institute for Population Research (BiB), \\ Friedrich-Ebert-Allee 4, Wiesbaden, 65185, Hesse, Germany.
}

Contributing authors: andreas.backhaus@bib.bund.de;

\begin{abstract}
In a recent contribution, Subramanian and Kumar (2021) state that increases in COVID-19 are unrelated to vaccination rates both across countries and across U.S. counties. I first discuss several empirical challenges related to the estimation of a meaningful relationship between these two variables which Subramanian and Kumar (2021) do not address. Using the same data sources, I then show that their findings disappear once the longitudinal character of the data is being utilized, underlining the arbitrary character of their result. Finally, I highlight that some of the county-level vaccination rates that Subramanian and Kumar (2021) cite as benchmarks for highly vaccinated locations are implausible and contradicted by additional data sources.
\end{abstract}

Keywords: COVID-19, Vaccination, United States

\section{Introduction}

In a recent contribution, [1] state that increases in COVID-19 are unrelated to vaccination rates both across countries and across U.S. counties. I first discuss several empirical challenges related to the estimation of a meaningful relationship between these two variables which [1] do not address. Using the same data sources, I then show that their findings disappear once the longitudinal 
Re: Subramanian and Kumar, Increases in COVID-19 are...

character of the data is being utilized, underlining the arbitrary character of their result. Finally, I highlight that some of the county-level vaccination rates that [1] cite as benchmarks for highly vaccinated locations are implausible and contradicted by additional data sources.

\section{Methods}

To begin with, the attempt to assess the effectiveness of vaccines by studying the association between the rate of new COVID-19 cases and the vaccination rate is challenging: Clearly, cases can occur both among the vaccinated and the unvaccinated population. The data sources on cases used by [1] do not differentiate case rates among the vaccinated population and case rates among the unvaccinated population. Given the high infectiousness of SARS-CoV-2 in general and of the Delta variant in particular, an increase in cases observed in locations with higher vaccination rates might be driven predominantly by the unvaccinated population. This would result in a spurious correlation between new cases and high vaccination rates. It is easy to conceive of such a setting: Locations with high vaccination rates could relax their COVID-19-related nonpharmaceutical interventions (NPIs) and their populations might behave less cautious due to the high vaccination coverage. Abstracting from immunity from prior infections, this response to high vaccination rates would expose the unvaccinated population to the virus more broadly, potentially resulting in more cases among the unvaccinated and hence more cases in these locations overall.

Closely related to this argument is the potential for reverse causality between new cases on the one hand and vaccination rates on the other hand. If such reverse causality existed, it would bias the estimated association between new cases and vaccinations. It is again easy to conceive of a situation in which reverse causality would exist: More individuals in a given location might decide to get vaccinated in response to a prolonged rise in the location's case count, which would result in a positive but spurious association between cases and vaccinations.

The data collection performed by [1] raises further questions. The first data source, which is country-level data from Our World In Data (OWID) [2], is originally available in longitudinal format, presenting repeated observations of both new cases and vaccination rates by country over time. [1] choose to reduce the data to a single cross-section by incorporating only the latest available vaccination rates and 7 -day-case rates into their analysis. The situation is similar regarding the data on cases and vaccinations in U.S. counties that [1] retrieve from the COVID-19 community profile reports published by the White House COVID-19 Team [3]. These reports have been published over several months, partially on a daily basis, providing a plethora of data. Still, [1] only use the latest publication as a cross-sectional data set in their analysis. While the decision to discard the additional data may already seem questionable in itself, a well-known shortcoming of cross-sectional analyses is that there might 
exist unobserved and time-invariant country- (or county-) specific factors that might be correlated with a country's (or county's) vaccination rates and case rates, resulting in biased estimates of the association between cases and vaccinations. Cross-sectional data cannot address this problem, while longitudinal data allow eliminating the bias from the unobserved heterogeneity by estimating fixed effects for each country (or county) for which multiple observations over time are available. Finally, the inclusion criteria chosen and applied by [1] to the OWID data result in several countries with high-capacity public health agencies being excluded from the sample, among them Belgium, France, Germany, the Netherlands, Spain, and the United Kingdom. Given that these countries have been publishing COVID-19-related data extensively throughout the pandemic, the reasons for them not meeting the inclusion criteria are likely idiosyncratic and potentially related only to the chosen cut-off date.

In the following, I demonstrate the arbitrariness of the association between COVID-19 cases and vaccination rates that [1] find by performing simple correlations between the two variables. Using the same data sources but making use of their longitudinal character, I show that the application of standard techniques in longitudinal regression analysis can result in negative and statistically significant associations between new cases and vaccination rates, which is opposite to the findings of [1]. Regarding the OWID data source, I incorporate all country-level case rates and vaccination rates from all time periods since the start of the first vaccination campaigns. In addition to using the full longitudinal data for the 68 countries considered by [1], I further extend the analysis to all countries for which case and vaccination data are provided by OWID. Regarding the county-level U.S. data, I combine thirteen of the COVID-19 community profile reports that have been published on the same day of the week between June 7 and August 30, 2021 into a longitudinal county-level data set of the new cases per 100,000 population reported in the last 7 days and the share of the population that is fully vaccinated. Note that the state of Texas is entirely missing from the community profile reports, as the state does not report county-level vaccination data to the CDC. The longitudinal data sets allow me to eliminate the potential bias from unobserved and time-invariant factors by regressing the COVID-19 case rates on the vaccination rates and fixed effects for each country (or county). In addition, common shocks to all observations in a given period can be controlled for by adding period fixed effects to the regression. I incorporate both approaches into my extension of the analysis performed by [1].

Finally, both the country-level and the county-level observations in the two data sets utilized by [1] are very heterogeneous in terms of their population size. While the population of the sample countries ranges from a few 100,000 to more than one billion, the population of the sample counties spans a few hundred to more than ten million. Disregarding these differences results in each observation receiving equal weight in the regression analysis irrespective of its population size. The regression results may therefore not accurately reflect the population-wide association between new COVID-19 cases and vaccination 
Re: Subramanian and Kumar, Increases in COVID-19 are...

rates. Therefore, I perform the regressions once without weighting and other time with weighting each observation by its population size, with the latter being provided directly by the referenced data sources.

\section{Findings}

Table 1 reports results on the association between new cases of COVID-19 and vaccination rates from using the country-level OWID sample. As reported in column 1, pooling all country-period observations and regressing the incidence of new COVID-19 cases per 1 million population on the full vaccination rates yields a positive and statistically insignificant association, similar to the cross-sectional result reported by [1]. However, adding country fixed effects to the regression changes the sign of the association and renders it weakly significant, as shown in column 2. Hence, estimating the association using only the variation in new cases and vaccination rates within the sample countries yields a result that is both quantitatively and qualitatively different from the pooled correlation. The association between new cases and vaccination rates remains negative and increases in magnitude if period fixed effects are added to the regression, as reported in column 3. Extending the OWID sample to all available countries and periods more than doubles the number of observations and still yields a negative association between new COVID-19 cases and the share of the fully vaccinated population, with the estimate indicating this association now being highly statistically significant (column 4). Qualitatively, a similar pattern is observed when the observations are weighted by the country population, as displayed in columns 5-8: The pooled regression indicates a positive and weakly significant association, which turns negative as soon as country fixed effects are included. The magnitude of the negative association, however, is larger than in the unweighted regressions when period fixed effects are added and the full OWID sample is being utilized.

The results from using the sample of U.S. counties are even more ambiguous, as reported in Table 2. Pooling all county-period observations yields a negative and insignificant association between new COVID-19 cases and the fully vaccinated population share (column 1). Adding county fixed effects then yields a large, highly significant, and positive association between cases and vaccinations (column 2). However, this positive association disappears again entirely in terms of magnitude and significance once period fixed effects are included (column 3). The U.S.-specific setting further allows adding interactions of the period fixed effects with state fixed effects to the regression in order to control for state-specific dynamics in new COVID-19 cases. This additional check has no impact on the association between new cases and vaccination rates in the unweighted regression reported in column 4 . Weighting the sample counties by population predominantly affects the magnitudes of the estimates while leaving their qualitative interpretation unchanged (columns 5-8), with the exception of the last column, where the addition of the interacted state and period fixed effects now renders the association negative and highly significant. 
Re: Subramanian and Kumar, Increases in COVID-19 are...

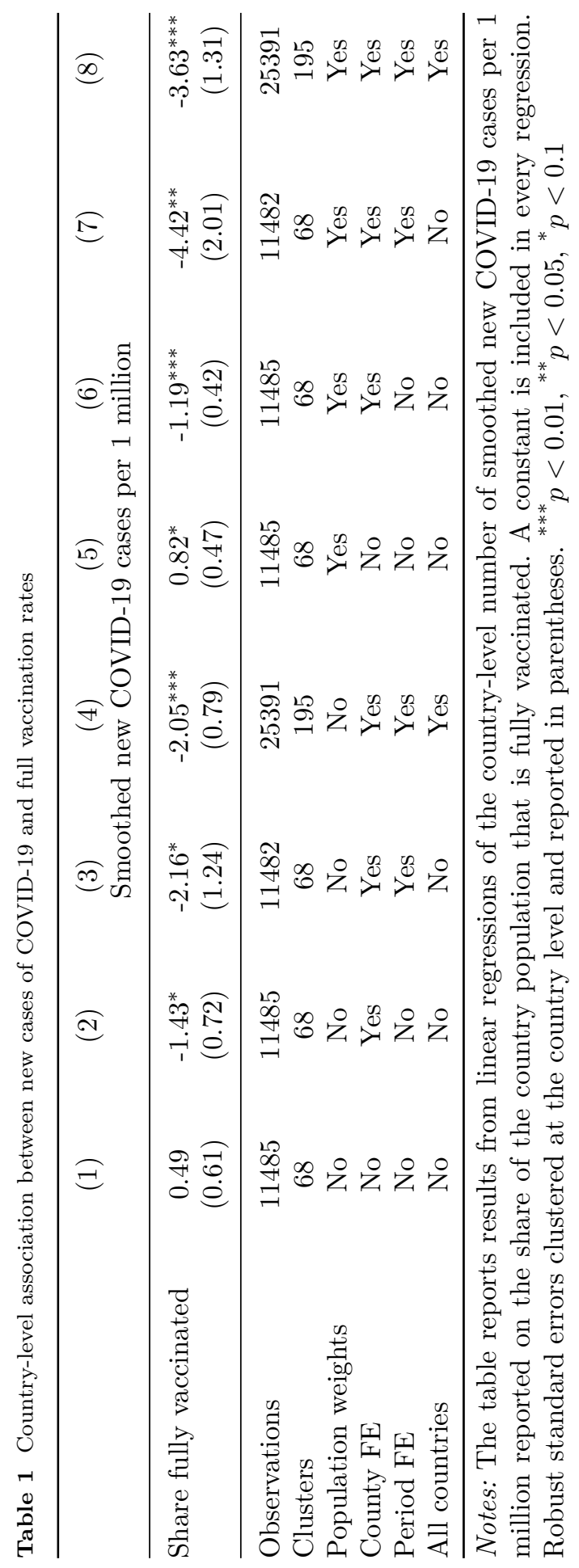


6 Re: Subramanian and Kumar, Increases in COVID-19 are...

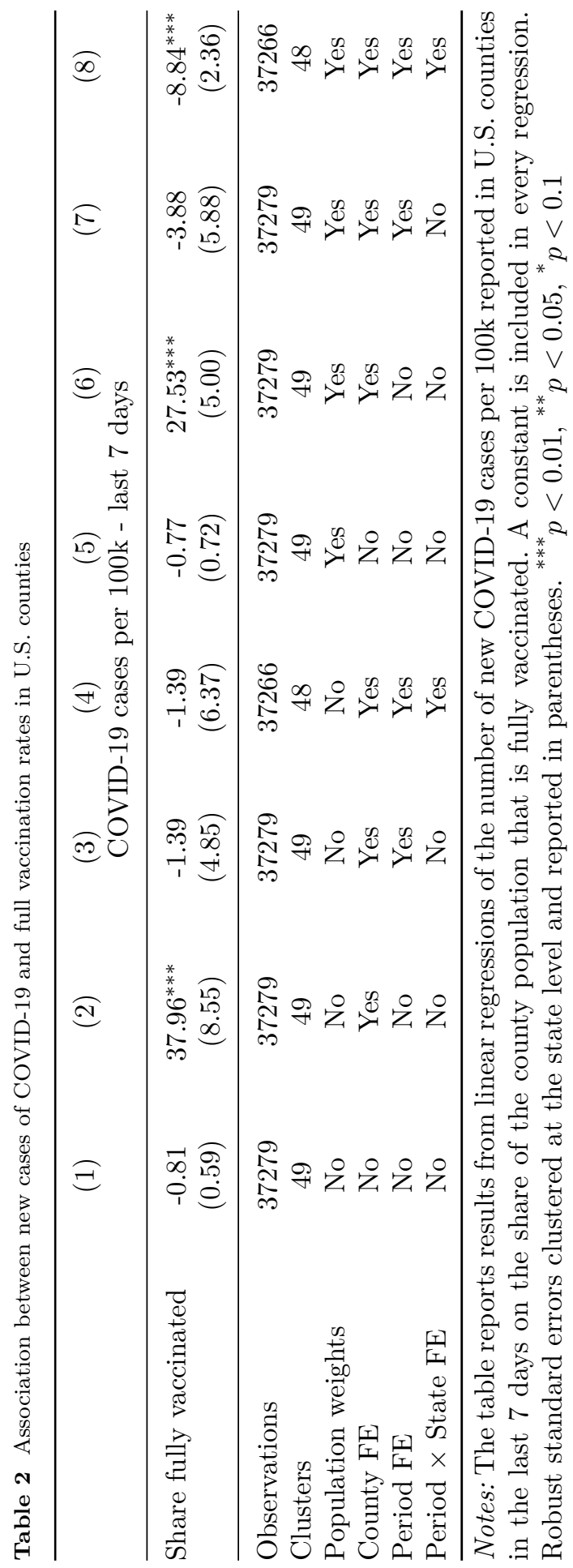


Finally, [1] state that "Of the top 5 counties that have the highest percentage of population fully vaccinated (99.9-84.3\%), the US Center for Disease Control and Prevention (CDC) identifies 4 of them as "High" Transmission counties." Specifically, they cite Chattahoochee (Georgia), McKinley (New Mexico), and Arecibo (Puerto Rico) as counties that have more than $90 \%$ of their population fully vaccinated while being classified as "High" transmission counties.

These proclaimed extremely high vaccination rates should immediately raise scepticism given that the COVID-19 vaccines are not approved yet for children of all ages. Hence, a full vaccination rate of $99.9 \%$ is technically impossible at the moment. Indeed, on closer inspection, the stated vaccination rates appear dubious: According to the data reported at the Vaccine Distribution Dashboard by the Georgia Department of Public Health [4], only 19.4\% of the residents of Chattahoochee county have been fully vaccinated by October 11, 2021. The nearby location of Fort Benning may severely inflate the CDC-reported number of doses administered in the county due to the vaccine mandate applying to U.S. military service members. Further, the New Mexico Department of Public Health reports on its COVID-19 Vaccine Dashboard that $85.4 \%$ of the residents of McKinley county have been fully vaccinated by October 11, 2021 [5]. While this figure already rejects the claim that more than $90 \%$ of the county's population are fully vaccinated, it needs to be further discounted, as it is based only on the county's population that is at least 18 years old. While the $99.9 \%$ full vaccination rate that the CDC reports for the county of Arecibo [6] cannot be immediately contrasted with a different figure, it should be noted that the CDC reports a population estimate of 81,966 for Arecibo, while simultaneously reporting that 91,617 vaccination series have been completed in Arecibo by October 11, 2021, raising doubts about the reported full vaccination rate.

\section{Conclusion}

Descriptive and superficial associations between aggregate case rates and vaccination rates can be highly misleading and sensitive to the treatment of the data, as argued and demonstrated above. Their scientific validity regarding the effectiveness of the COVID-19 vaccines should therefore be regarded as being severely constrained. Consequently, these associations can provide only very limited guidance regarding the public health actions that may be chosen to complement the ongoing vaccination campaigns.

\section{Declarations}

- Funding: No external funding has been received for the writing of this manuscript.

- Conflict of interest/Competing interests: The author declares no conflict of interest and no competing interests.

- Ethics approval: Not applicable.

- Consent to participate: Not applicable. 
- Consent for publication: Not applicable.

- Availability of data and materials: All data used in this manuscript are publicly available at the referenced sources.

- Code availability: The code for the presented analyses is available from the author on request.

- Authors' contributions: Andreas Backhaus is the sole author of the manuscript. The views presented in this manuscript are those of the author and do not necessarily reflect the views of the Federal Institute for Population Research.

\section{References}

[1] Subramanian, S.V., Kumar, A.: Increases in covid-19 are unrelated to levels of vaccination across 68 countries and 2947 counties in the united states. European Journal of Epidemiology (2021)

[2] Ritchie, H., Mathieu, E., Rodés-Guirao, L., Appel, C., Giattino, C., Ortiz-Ospina, E., Hasell, J., Macdonald, B., Beltekian, D., Roser, M.: Coronavirus pandemic (COVID-19). Our World in Data (2020)

[3] White House COVID-19 Team: COVID-19 community profile report (2021). https://healthdata.gov/Health/ COVID-19-Community-Profile-Report/gqxm-d9w9

[4] Georgia Department of Public Health: Vaccine Distribution Dashboard (2021). https://experience.arcgis.com/experience/ 3d8eea39f5c1443db1743a4cb8948a9c

[5] New Mexico Department of Health: COVID-19 Vaccine Dashboard (2021). https://cvvaccine.nmhealth.org/public-dashboard.html

[6] Centers for Disease Control and Prevention: CDC COVID Data Tracker - COVID-19 Integrated County View (2021). https://covid.cdc.gov/ covid-data-tracker/\#county-view 